\title{
Persistent HIV-infected cells in cerebrospinal fluid are associated with poorer neurocognitive performance
}

\author{
Serena Spudich, ${ }^{1}$ Kevin R. Robertson, ${ }^{2}$ Ronald J. Bosch, ${ }^{3}$ Rajesh T. Gandhi, ${ }^{4}$ Joshua C. Cyktor, ${ }^{5}$ Hanna Mar, ${ }^{3}$ \\ Bernard J. Macatangay, ${ }^{5}$ Christina M. Lalama, ${ }^{3}$ Charles Rinaldo, ${ }^{5}$ Ann C. Collier, ${ }^{6}$ Catherine Godfrey, ${ }^{7}$ Joseph J. Eron, ${ }^{2}$ \\ Deborah McMahon, ${ }^{5}$ Jana L. Jacobs, ${ }^{5}$ Dianna Koontz, ${ }^{5}$ Evelyn Hogg, ${ }^{8}$ Alyssa Vecchio, ${ }^{2}$ and John W. Mellors ${ }^{5}$ on behalf of the AIDS \\ Clinical Trials Group HIV Reservoirs Cohort Study ${ }^{9}$ \\ ${ }^{1} Y a l e$ University, New Haven, Connecticut, USA. ${ }^{2}$ University of North Carolina at Chapel Hill, Chapel Hill, North Carolina, USA. ${ }^{3}$ Harvard TH Chan School of Public Health, Boston, Massachusetts, USA \\ ${ }^{4}$ Massachusetts General Hospital, Boston, Massachusetts, USA. ${ }^{5}$ University of Pittsburgh, Pittsburgh, Pennsylvania, USA. ${ }^{6}$ University of Washington, Seattle, Washington, USA. ${ }^{7}$ NIH, Bethesda, Maryland, \\ USA. ${ }^{8}$ Social \& Scientific Systems, Silver Spring, Maryland, USA. ${ }^{9}$ The AIDS Clinical Trials Group HIV Reservoirs Cohort Study group is detailed in the Supplemental Acknowledgments.
}

BACKCROUND. Persistence of HIV in sanctuary sites despite antiretroviral therapy (ART) presents a barrier to HIV remission and may affect neurocognitive function. We assessed HIV persistence in cerebrospinal fluid (CSF) and associations with inflammation and neurocognitive performance during long-term ART.

METHODS. Participants enrolled in the AIDS Clinical Trials Group (ACTC) HIV Reservoirs Cohort Study (A5321) underwent concurrent lumbar puncture, phlebotomy, and neurocognitive assessment. Cell-associated HIV DNA and HIV RNA (CA-DNA, CA-RNA) were measured by quantitative PCR (qPCR). in peripheral blood mononuclear cells (PBMCs) and in cell pellets from CSF. In CSF supernatant and blood plasma, cell-free HIV RNA was quantified by qPCR with single copy sensitivity, and inflammatory biomarkers were measured by enzyme immunoassay.

\begin{abstract}
RESULTS. Sixty-nine participants ( $97 \%$ male, median age 50 years, CD4 696 cells $/ \mathrm{mm}^{3}$, plasma HIV RNA <100 copies/mL) were assessed after a median 8.6 years of ART. In CSF, cell-free RNA was detected in 4\%, CA-RNA in 9\%, and CA-DNA in 48\% of participants (median level 2.1 copies $/ 10^{3}$ cells). Detection of cell-free CSF HIV RNA was associated with higher plasma HIV RNA $(P=0.007)$. CSF inflammatory biomarkers did not correlate with HIV persistence measures. Detection of CSF CA-DNA HIV was associated with worse neurocognitive outcomes including global deficit score $(P=\mathbf{0 . 0 0 5})$, even after adjusting for age and nadir CD4 count.
\end{abstract}

CONCLUSION. HIV-infected cells persist in CSF in almost half of individuals on long-term ART, and their detection is associated with poorer neurocognitive performance.

FUNDING. This observational study, AIDS Clinical Trials Group (ACTC) HIV Reservoirs Cohort Study (A5321), was supported by the National Institutes of Health (NIAID and NIMH).

\section{Introduction}

In persons living with HIV, the persistence of intact proviruses integrated within host cellular DNA despite long-term antiretro-

\section{Related Commentary: p. 3052}

\section{Authorship note: SS and KR are co-primary authors.}

Conflict of interest: SS and KRR codirect a clinical trial that provides study medication donated by Viiv Healthcare. CG wrote this paper in her capacity as an NIH employee, but the views expressed in this paper do not necessarily represent those of the NIH. JWM is a consultant for Gilead and Merck, and owns share options in Co-Crystal Pharmaceuticals, Inc. EH is employed by a commercial company, Social \& Scientific Systems. JJE is a consultant to Gilead Sciences, Merck \& Co, Janssen, and ViiV Healthcare. KRR consulted for ViiV Healthcare. ACC is a member of a Data Safety and Monitoring Board for studies sponsored by Merck \& Co. Copyright: @ 2019, American Society for Clinical Investigation. Submitted: January 15, 2019; Accepted: May 14, 2019; Published: July 15, 2019. Reference information: J Clin Invest. 2019;129(8):3339-3346. https://doi.org/10.1172/JCl127413. viral therapy (ART) remains a barrier to curing the infection (1). While most research has focused on blood $\mathrm{CD} 4^{+}$memory $\mathrm{T}$ cells as the major viral reservoir, HIV persists in other sites, including mucosal lymphoid tissue, bone marrow, and the brain (2-4). HIV infects the brain soon after infection and has a number of CNS manifestations including HIV-associated neurocognitive disorder (HAND), which range in severity from severe HIV-associated dementia (HAD) to the more subtle and prevalent mild neurocognitive (MND) and asymptomatic neurocognitive impairment (ANI) (5-7). Although neurological complications of HIV have been well recognized, questions remain about persistence of virus in the CNS that are difficult to address given the inaccessibility of brain tissue in living individuals.

Cerebrospinal fluid (CSF) offers a window into neuropathogenesis of HIV in living individuals; most prior studies, however, have focused on analysis of soluble biomarkers or measurement of cell-free HIV RNA in CSF supernatant. Multiple studies have revealed persistently abnormal levels of soluble inflammatory 
molecules in CSF during suppressive ART, as well as correlations between CSF inflammation, CSF biomarkers of active neuronal injury, and clinical measures of neurocognitive performance, supporting the utility of quantitation of CSF biomarkers (8-9). Assessment of CSF HIV RNA has been restricted during ART since it is often below the limit of standard assays, though there are reports of detectable HIV RNA in CSF despite suppression in plasma in a minority of asymptomatic individuals, termed asymptomatic CSF HIV escape (10-12). A limited number of studies of the cellular component of CSF suggest that CSF cellular profiles largely reflect those in the blood (13-15) and that, in untreated individuals, HIV DNA may be detected in CSF cells (16-17). To what extent HIV persists in CSF cells in people on long-term effective ART is uncertain.

In addition to the importance of evaluating HIV in the CSF to inform cure research, there are gaps in our knowledge regarding whether HIV persistence is associated with HAND. CNS HIV infection can result in impaired neurocognitive performance, which manifests as a HAND in $15 \%$ to $55 \%$ of participants and remains a major cause of morbidity in the ART era $(6,18)$. Although the incidence of HAND has decreased with the introduction of ART, the milder forms of HAND persist and interfere with daily activities (18). As the population with HIV ages, the prevalence and severity of HAND are of concern, particularly since there is uncertainty around the pathogenesis of the continued neurocognitive decline. The progression of neurologic disease in otherwise well-controlled HIV infection has also been studied in the context of symptomatic CSF escape in which there is a CSF/plasma HIV RNA discordance associated with the development of new neurologic symptoms (19).

To gain further insights into HIV persistence on ART, we assayed CSF fluid and cells with sensitive qPCR detection methods for cell-free HIV RNA and cell-associated HIV DNA and HIV RNA (CA-DNA, CA-RNA) and evaluated associations with blood HIV measures and soluble CSF biomarkers of immune activation in individuals with sustained plasma viral suppression. Clinical significance of the laboratory measures was evaluated by neuropsychological assessments to examine whether neurocognitive performance is associated with the persistence of HIV or inflammatory markers in the CSF.

\section{Table 1. Baseline characteristics of study participants}

\begin{tabular}{|c|c|c|}
\hline Characteristic & $\mathrm{HIV}^{+}(n=69)$ & HIV- $^{-}(n=19)$ \\
\hline Age, years & $50(45-56)$ & $49(38-56)$ \\
\hline Sex, proportion (\% male) & $67 / 69(97)$ & $13 / 19(68)$ \\
\hline Duration of ART, years & $8.6(7.4-10.4)$ & -- \\
\hline Blood CD4 ${ }^{+} T$ cell count at lumbar puncture, cells $/ \mathrm{mm}^{3}$ & $696(502-906)$ & $770(701-934)$ \\
\hline CD4/CD8 ratio at lumbar puncture & $1.0(0.8-1.2)$ & $1.8(1.4-2.5)$ \\
\hline Plasma HIV RNA $<40 \mathrm{cps} / \mathrm{mL}$ at lumbar puncture, proportion (\%)* & $67 / 69(97)$ & -- \\
\hline CSF WBC, cells/ $\mu \mathrm{L}$ & $1(0-2)$ & $1(0-3)$ \\
\hline CSF cell equivalents detected, no. CCR5 copies & $7903(4179-21700)$ & -- \\
\hline CSF/plasma albumin ratio & $5.3(3.8-7.0)$ & $5.0(3.2-6.8)$ \\
\hline
\end{tabular}

*Plasma HIV RNA values in 2 participants with greater than or equal to 40 copies $/ \mathrm{mL}$ were 63 copies $/ \mathrm{mL}$ and 76 copies $/ \mathrm{mL}$. All values are median and interquartile range (Q1, Q3) unless indicated.

\section{Results}

Characteristics of study participants. Sixty-nine participants with HIV (97\% male, $71 \%$ white) with median age 50 years (range 26 to 72 years), CD $4^{+} \mathrm{T}$ cell count $696 / \mathrm{mm}^{3}$ (range 240 to 1664 ), and median ART duration of 8.6 years (range 5.4 to 16.4 years) initiated during chronic HIV infection contributed paired CSF and blood samples. All had plasma HIV RNA of less than 40 copies/ $\mathrm{mL}$ at the time of CSF sampling except 2 participants (with 63 and 76 copies $/ \mathrm{mL}$, respectively). For comparison, paired CSF and blood samples were collected from 19 HIV-negative participants with a median age of 49 years old, $68 \%$ male, and median CD4 ${ }^{+}$ T cell count of $770 / \mathrm{mm}^{3}$ (Table 1 ).

Sixty-five of 69 participants, all with at least 12 years of education, participated in a 7-domain neuropsychological test battery resulting in a global deficit score (GDS) and total $Z$ score.

Detection of persistent HIV-1 in CSF and blood. HIV DNA was detected in CSF cell pellets (centrifuged from $\sim 13 \mathrm{~mL} \mathrm{CSF}$ ) in $48 \%$ of participants (95\% CI: $36 \%-60 \%$ ). By contrast, $9 \%$ (95\% CI: $3 \%-18 \%$ ) had detectable CSF CA-RNA and only $4 \%$ (95\% CI: 1\%-12\%) had detectable HIV RNA in 3-5 mL cell-free CSF fluid (Figure 1A).

Median levels of detectable CA-DNA in CSF were 2.1 copies/ $10^{3}$ (range $0.12-7.00$ copies $/ 10^{3}$ ) CSF cells. Limiting dilution analysis of PBMC samples from this study revealed that the number of cells assayed for HIV DNA did not affect the quantification of HIV DNA (Supplemental Table 1; supplemental material available online with this article; https://doi. org/10.1172/JCI127413DS1). Levels of CA-DNA in PBMCs are typically reported per million $\mathrm{CD} 4^{+} \mathrm{T}$ cells rather than per thousand cells, giving a median of 548 copies $/ 10^{6}$ (range 0.3-6553 copies $/ 10^{6}$ ) $\mathrm{CD}^{+} \mathrm{T}$ cells in the current study. The median value of CA-DNA expressed per one thousand $\mathrm{CD} 4^{+}$ $\mathrm{T}$ cells is 0.5 copies, compared with 2.1 CA-DNA copies per thousand total cells in CSF. Although comparisons across blood fluids containing different cell types is complex, our findings suggest that the frequency of CSF cells containing CA-DNA may be similar to or even higher than those in circulating PBMCs. Indeed, limiting dilution analysis of PBMCs from a subset of individuals in the current study shows that the number of copies of HIV DNA detected normalized for cell input was the same or higher in CSF cells than in PBMCs (Supplemental Table 1).

Among the 33 participants with detectable CSF HIV DNA, 6\% and 6\% had detectable CSF CA-RNA and cell-free HIV RNA, respectively. Participants with detectable cell-free HIV RNA in CSF had higher levels of plasma HIV RNA by integrase single copy assay (iSCA) than those without detectable CSF HIV RNA (median plasma VL, 5.9 vs $<0.4 \mathrm{cps} / \mathrm{mL}, P=0.007)$. By contrast, detection of CA-DNA in CSF was not associated with HIV DNA levels in PBMCs nor with detection of plasma HIV RNA by iSCA. Importantly, pre-ART CD4 count $(P=0.27)$, pre-ART plasma HIV RNA level $(P=0.15)$, pre-ART CD4/CD8 ratio $(P=0.09)$, and years of ART at time of lumbar puncture $(P=0.95)$ were not significantly associated with detection of CSF HIV DNA (Figure 1, B-E). 
A

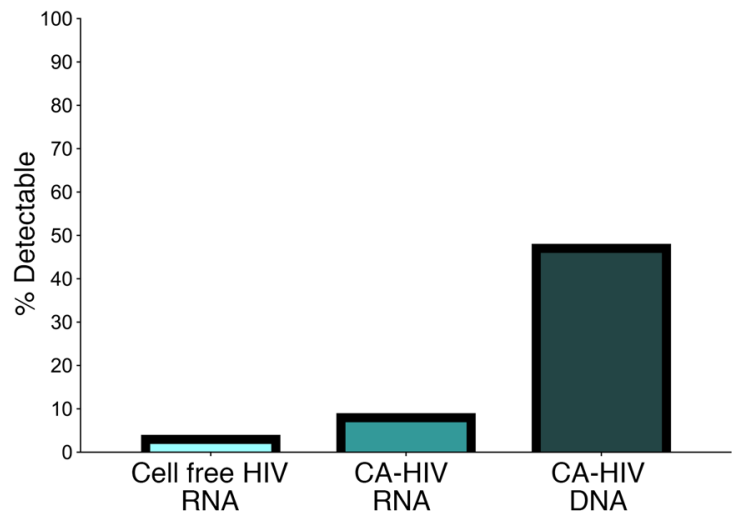

B

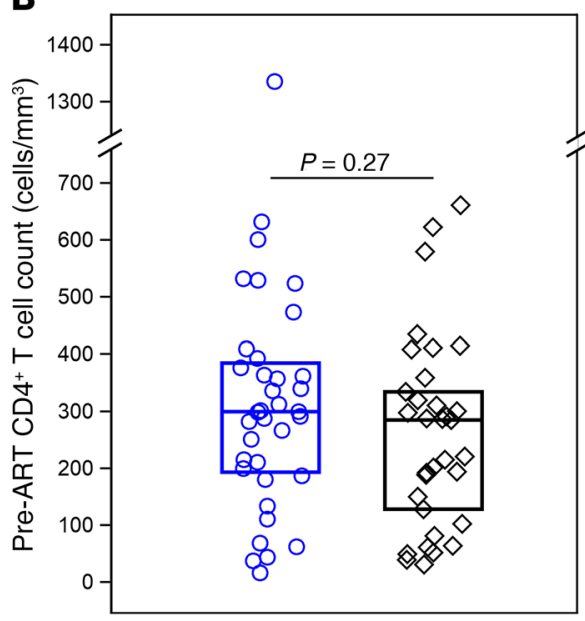

D

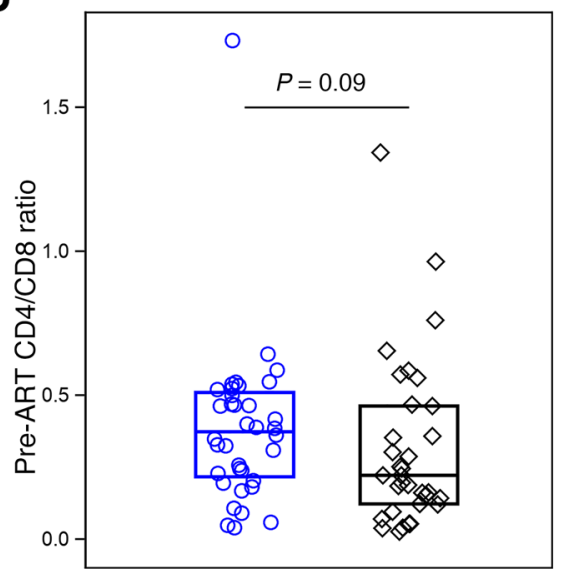

C

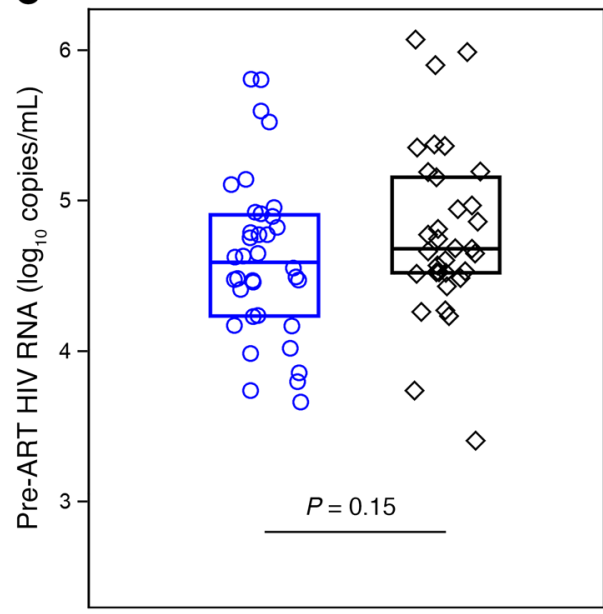

$\mathbf{E}$

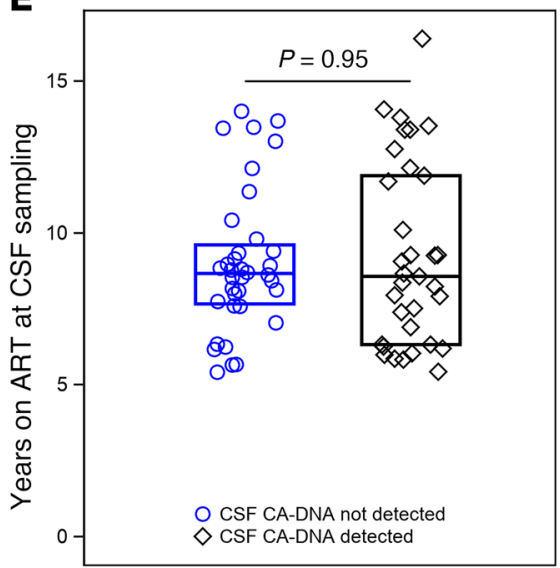

Figure 1. HIV persistence measures in CSF during ART. In CSF of individuals on sustained ART started in chronic infection, cell-free HIV RNA was detected in 4\% (95\% Cl: $1 \%-12 \%)$, CA-HIV RNA was detected in $9 \%$ (95\% Cl: 3\%-18\%), and CA-HIV DNA was detected in 48\% (95\% Cl: 36\%-60\%). The boxes indicate percentage positive (A). Detection of CA-HIV DNA in CSF cells did not significantly associate with pre-ART CD4 count (B), HIV RNA level (C), CD4/CD8 ratio (D), or years on ART (E), where the boxes indicate median values.

biomarkers: negative with IL-6 (Spearman $=-0.38, P=0.001)$, and positive with CXCL-10 (Spearman $=0.44$, $P<0.001)$, sCD14 (Spearman $=0.39$, $P=0.001$ ), and sCD163 (Spearman = $0.33, P=0.006$ ). Inflammatory biomarkers, especially indicators of myeloid cell activation, also correlated between CSF and plasma. However, CSF inflammatory biomarkers did not correlate with CSF CA-DNA, CA-RNA, or cell-free HIV RNA. CSF sCD14 and sCD163 were associated with higher levels of plasma HIV RNA $(r=0.34, P=0.004 ; r=0.29$, $P=0.017)$. CSF CA-DNA and plasma inflammatory markers were not statistically associated. Levels of CSF inflammatory biomarkers correlated with one another and with plasma levels in the $\mathrm{HIV}^{+}$participants, regardless of whether they had detected CSF HIV DNA. Higher CSF neopterin, CXCL-10, CCL-2, sCD14, and sCD163 correlated with older age $(P \leq 0.016)$; higher CSF neopterin correlated with lower pre-ART CD4/ CD8 ratio $(r=-0.29, P=0.017$; Figure 2, C and D, and Supplemental Table 3).

Association between CSF HIV-1 DNA and neurocognitive performance. A critical element of our study was the inclusion of neurocognitive testing to assess for relationships between markers of HIV persistence and clinical status. The median neurocognitive total $Z$ score was 0.2 (range -1.1-1.5), and median GDS was 0.2 (range 0.0-1.3). Detection of CSF HIV DNA (versus no detection) was significantly associated with poorer neurocognitive total $Z$ score $(P=0.044$, Wilcoxon) and GDS ( $P=0.005$; Figure 3$)$. The association between detectable CSF HIV DNA and neurocognitive dysfunction persisted after adjusting for pre-ART CD4 count, current CD4 count and age. Fine motor, executive functioning, and attention GDS were particularly impacted (data not shown). Soluble biomarkers of immune activation in CSF were not associated with neurocognitive performance (data not shown). Among those with no detectable CSF HIV DNA, 4 of

Biomarkers of inflammation and immune activation in CSF and blood. Consistent with previous reports, we found that 2 CSF inflammatory markers (sCD163 and CXCL-10) were elevated in $\mathrm{HIV}^{+}$ versus HIV $\mathrm{HV}^{-}$participants despite prolonged ART (Figure 2, A and B, and Supplemental Table 2). Within $\mathrm{HIV}^{+}$participants, we observed associations between CSF neopterin and other CSF inflammatory
35 (11\%) were clinically impaired while 9 of 30 (30\%) with detectable CSF HIV DNA were in the clinically impaired range (GDS $\geq 0.5)$ (20).

\section{Discussion}

The striking observation that almost half of participants harbored HIV-infected cells in the CSF demonstrates that the CNS compart- 

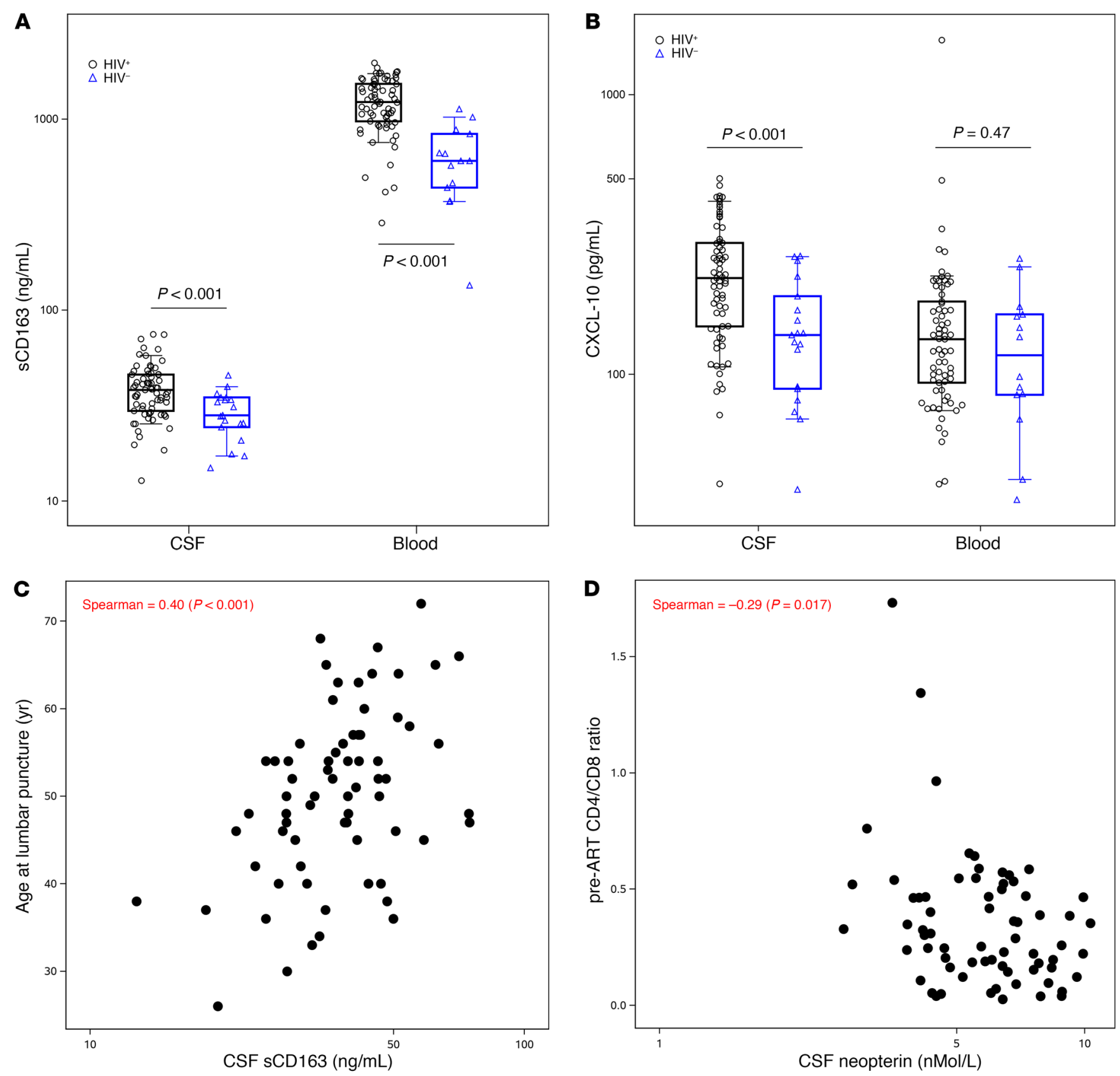

Figure 2. Soluble inflammatory biomarkers in CSF. CSF sCD163 (A) and CXCL-10 (B) were elevated in CSF in $\mathrm{HIV}^{+}$compared with HIV- participants. Age at CSF collection associated with levels of multiple biomarkers including sCD163 (C), and lower pre-ART CD4/CD8 ratio predicted higher CSF neopterin (D). Segment inside the box indicates median, box edges represent 25 th and 75 th percentiles and whiskers 10th and 90th percentiles.

ment is a site of viral persistence despite many years of viremia suppression on ART. A recent study showed persistence of HIV DNA in CSF cells in 10 of 16 participants after ART suppression for a median of 2.6 years, including 2 who started ART during primary infection (21). Our study now reveals frequent detection of HIV DNA in CSF in a larger cohort of individuals $(N=69)$ who initiated ART during chronic infection and were suppressed and on ART for a median of 8.6 years. Older studies from the pre-ART era using previous PCR techniques have shown that HIV proviral DNA could be measured in CSF cells in $68 \%$ to $87 \%$ of untreated individuals, and suggest that a higher proportion of cells may harbor HIV DNA in CSF than in PBMCs $(16,22-23)$. Large studies of CSF cells in participants on ART who have suppressed plasma HIV RNA levels have not been previously performed. Our study indicates that examination of CSF cells is important in assessing residual HIV in compartments during ART.

We found low rates of detectable HIV RNA in the cell-free CSF fraction (4\% of samples) and within CSF cell pellets (9\% of samples), suggesting low levels of HIV transcription within cells and infrequent release into the extracellular space during systemically suppressive ART. A lower rate of detection of HIV RNA in cell-free CSF in our study compared with $42 \%$ CSF HIV RNA detected by iSCA methods in 220 treated individuals in a recent study of CNS HIV-1 Antiretroviral Therapy Effects Research (CHARTER) par- 


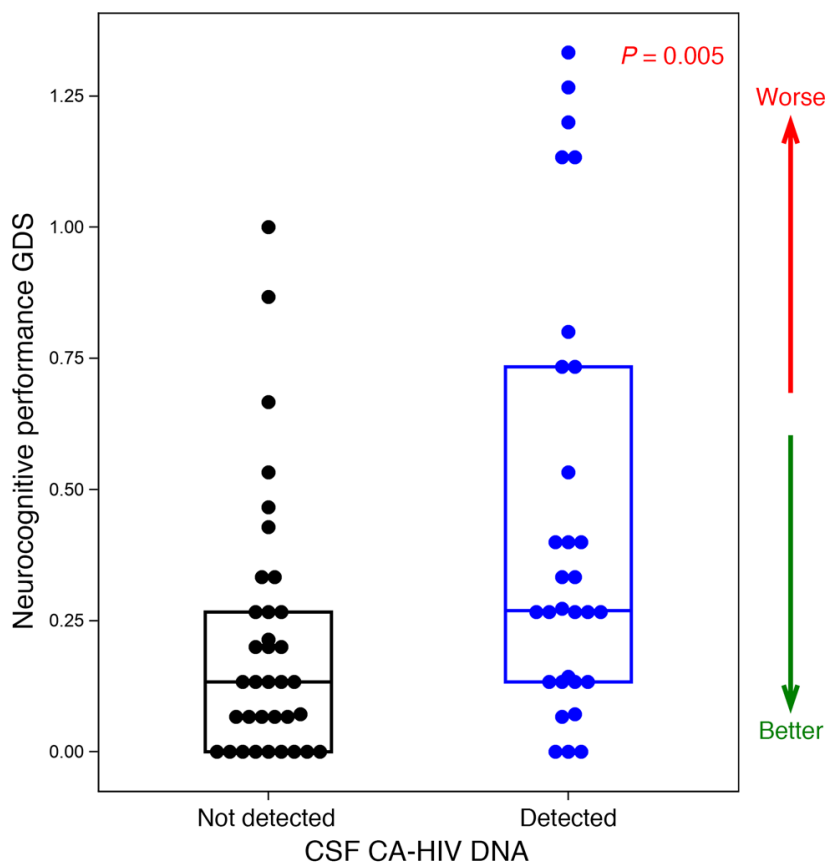

Figure 3. Association between detection of CSF CA-HIV DNA and neuropsychological performance. GDS, global deficit score. Segment inside the box indicates median, box edges represent 25th and 75th percentiles.

ticipants may be due to a number of factors (24). Given the fact that our cohort was enrolled and followed long-term within ACTG studies with documented viral suppression for numerous visits, it is likely that our cohort was more consistently well-suppressed than the participants in the CHARTER study who were identified by a single plasma HIV RNA less than or equal to 50 copies $/ \mathrm{mL}$. Participants in our cohort were also more likely to be on their first regimen ( $42 \%$ of our study participants versus only $22 \%$ of the CHARTER study participants), had been on ART for a longer duration (median 8.6 years in our study versus 5.8 years in the CHARTER study), and started ART at higher CD4 counts (median preART CD4 ${ }^{+}$count of 288 cells $/ \mathrm{mm}^{3}$ in our study versus 150 cells/ $\mathrm{mm}^{3}$ in the CHARTER study). All of these factors could plausibly explain the differences observed between the studies. The lower frequency of detection of cell-free HIV-1 RNA in the CSF in our study $(4 \%)$ compared with the CHARTER study $(42 \%)$ is unlikely to be due to differences in assay sensitivity because both assays have been validated to $1 \mathrm{copy} / \mathrm{mL}$ and similar volumes of CSF were assayed ( $5 \mathrm{~mL}$ in the current study versus $2 \mathrm{~mL}$ in the CHARTER study) (24-26). In addition, 2 prior studies using real-time qPCR assays with single copy sensitivity similar to that in the current study reported detectable HIV RNA in cell-free CSF supernatant at frequencies closer to those observed in our study: $6 \%$ to $14 \%$ of participants had detectable cell-free HIV RNA in CSF during ART $(27,28)$. Taken together, it is likely that differences between the CHARTER and current study populations, including the consistency and duration of viral suppression on ART, contributed to the lower frequency of detection of cell-free HIV-1 RNA detection in CSF reported here.

We do not know the origin of the HIV-infected cells in CSF. Mononuclear cells detected in CSF (lymphocytes, monocytes,
B cells, and NK cells) are believed to originate from the bone marrow and presumably reach the CSF via transmigration through blood-brain and blood-CSF barriers, or from "drainage" after immune surveillance of the brain and meninges, though a recent study suggests that CSF contains cells similar to brain-resident microglia, suggesting a possible brain origin of a subset of CSF cells (29). Our finding that HIV DNA is detected in a large proportion of CSF samples may indicate continuous trafficking of HIVinfected cells into the CSF from the periphery, where CA-HIV DNA is almost always detectable in $\mathrm{CD}^{+} \mathrm{T}$ cells during ART. However, in our study, concentrations of HIV DNA in blood $\mathrm{CD} 4^{+} \mathrm{T}$ cells were not significantly higher in those with HIV DNA detected in CSF. An alternative possibility is that CSF CA-HIV DNA may reflect release of infected cells into the CSF from the brain or meninges, which would suggest previously unrecognized sources of persistent HIV.

We cannot accurately compare the quantity of CA-HIV DNA in total CSF cells to those in blood $\mathrm{CD} 4^{+} \mathrm{T}$ cells in the present study nor in prior studies of blood or lymph nodes given the disparate cellular populations assessed. However, our limiting dilution assays suggested that quantitation of CA-HIV DNA in CSF was not substantially distorted by the low numbers of cells analyzed. Given this, the concentration of CA-HIV DNA in CSF samples appears to be comparable or higher to levels in PBMCs. The levels of CA-HIV DNA in brain tissue during ART are still not well quantified because few brain autopsy samples have been analyzed for persistent HIV from individuals who were taking virally suppressive ART or who did not have other confounding health conditions at the time of death $(30,31)$.

Though we have identified HIV-infected CSF cells in a high proportion of individuals on effective ART, the significance of this HIV DNA detection in relation to a persistent reservoir of replication-competent HIV in the CNS compartment is unknown. The genetic relatedness of cell-associated and cell-free virus in the CSF with that in blood has not been characterized. Further, the proportion of CA-HIV DNA in CSF cells which is intact or inducible is unknown. The latter is a fundamental unanswered question that has major implications for prevention and treatment of HIV-associated CNS disease as well as efforts for long-term HIV remission. Studies collecting larger CSF volumes to yield enough cells or development of novel assays for intact virus that use fewer cells may help to answer these important questions.

Previous studies have demonstrated associations between HIV persistence and markers of immune activation or inflammation in blood and other tissues $(32,33)$. Our finding that CSF levels of inflammatory biomarkers did not associate with CSF measures of HIV persistence is consistent with the lack of association between markers of HIV persistence and immune activation or inflammation in blood in a prior study of individuals on long-term effective ART in the ACTG HIV Reservoirs Cohort Study (A5321), from which the current study participants were enrolled (34). Though we did find associations between $2 \mathrm{CSF}$ markers of myeloid immune activation (sCD14 and sCD163) and plasma viremia, in this cohort of continuously virally suppressed individuals, viral persistence does not appear to be a main driver of chronic immune activation within compartments, nor is the latter driving viral persistence. Other causes 
of chronic immune activation and inflammation in HIV infection during ART could include immune changes and inflammation triggered in pre-ART HIV infection, chronic microbial translocation, effects of unrecognized coinfections, or viral persistence in tissues not accessed through the sampled fluids.

Neurocognitive impairment in individuals with HIV is likely multifactorial, with potential contributions from a variety of etiologies including persistent HIV infection, age, unresolved neuroinflammation, and comorbid conditions such as substance use, vascular dysfunction, mood disorders, and poor educational attainment (35-37). Despite this, we identified a significant association between detectable HIV DNA in CSF cells and neurocognitive performance, revealing potential clinical relevance of this viral measure in the CNS. It is possible that persistent HIV in the CNS may drive neurocognitive injury, though the mechanisms of this are unknown. Alternately, this may indicate a legacy effect, in which processes associated with long-term exposure to HIV prior to initiation of ART lead to both irreversible neurological injury and more extensive infection of cells that persist in CSF. Lack of an association with inflammatory biomarkers suggested that current inflammation does not lead to present neurocognitive dysfunction, but does not rule out prior inflammation as the underlying cause of neuronal injury. These findings underscore the potential clinical significance of HIV persistence in the CNS, which needs additional evaluation. Although the median neurocognitive performance in both groups in this study was within normal limits, $30 \%$ of those with detectable CSF CA-HIV DNA had neuropsychological performance in the clinically impaired range compared with $11 \%$ of those without CSF CA-HIV DNA. This difference in the rate of impairment between the groups implies an increased risk of HAND among those with detectable CSF CA-HIV DNA and calls for further investigation into the clinical consequence of persistent HIV in the CNS.

The novelty of the current study centers around its focus on measurement of HIV within the cellular component of CSF in participants with very long-term and well-documented plasma HIV RNA suppression on ART. Prior flow cytometry studies of CSF from $\mathrm{HIV}^{+}$participants have characterized phenotypes of CSF immune cells subsets $(14,15)$. CSF mononuclear cells are primarily composed of $\mathrm{T}$ lymphocytes in $\mathrm{HIV}^{-}$individuals and individuals with HIV pre- and post-ART (14). The CD $4^{+} \mathrm{T}$ cell percentage ranges from approximately $40 \%$ in participants who started ART during acute HIV infection to $25 \%$ in those who start ART in chronic infection (38), and CD4/CD8 ratio measured in blood by clinical labs highly correlates with the percentage of $\mathrm{CD} 4^{+} \mathrm{T}$ lymphocytes in CSF $(13,14)$.

A longitudinal analysis of people with HIV on suppressive therapy with both CSF and blood assessments to establish whether detection of HIV-infected cells persists over time and remains associated with neurocognitive function would add weight to our findings. Similarly, assessment of individuals who initiated ART during acute infection may be able to address questions about the legacy effect of HIV infection. Paradoxically, detection of HIV in CSF may be higher in those with early initiation of ART due to greater preservation of $\mathrm{CD}^{+} \mathrm{T}$ cells within the CSF. Additional studies should compare associations between ART regimens and the levels of CSF HIV DNA and evaluate the longitudinal relationship between HIV persistence in CSF and neurocognitive function.

Overall, persistence of HIV in the CNS may represent a barrier to optimal neurocognitive function and in addition provide an obstacle to the cure for HIV. The sensitive methods of HIV detection described in the current study, combined with more detailed analyses of the proviruses in infected cells found in the CSF, can provide new insight into the CNS reservoir of HIV and how best to eradicate or control it.

\section{Methods}

Study participants and clinical procedures. Individuals with HIV who started ART during chronic infection enrolled in the longitudinal ACTG HIV Reservoirs Cohort Study (A5321) and consented to an optional single cross-sectional lumbar puncture, concurrent blood draw, and neuropsychological assessment as part of the approved A5321 study protocol. Participants initiated ART during ACTG trials for treatment-naive persons and were followed at least every 6 months while continuing to receive ART. As a result, clinical data and paired plasma and PBMC samples were available from pre-ART study visits. In addition, consistent documentation of ART status and HIV viral loads was available for these participants from the time of treatment initiation throughout long-term follow-up. A5321 participants enrolled in this study had plasma viral suppression (HIV RNA levels $\leq 50$ copies $/ \mathrm{mL}$ ) starting at week 48 after initiating ART and had sustained viral suppression at follow-up time points (no consecutive HIV RNA levels $>50$ copies $/ \mathrm{mL}$ ); there were no ART interruptions for 21 days or longer in the 4 years prior to the LP.

CSF and blood samples from HIV participants of a similar median age to the A5321 participants were available from participants in the IRB-approved HIV Associated Reservoirs and Comorbidities (HARC) study at Yale University for analysis of soluble immune activation biomarkers for comparison to the HIV samples. These HIV comparison participants were individuals who were recruited from the local New Haven community as controls for HIV studies, had no known neurological conditions, and who participated in blood draw and lumbar punctures for research purposes only.

Lumbar punctures were performed using 22 gauge pencil-point needles in most cases to withdraw 22 to $25 \mathrm{~mL}$ of CSF. CSF samples were promptly centrifuged and supernatants and cell pellets stored at $-80^{\circ} \mathrm{C}$ according to established ACTG CSF processing protocols.

Laboratory methods. HIV persistence was measured as CA-HIV DNA and RNA and cell-free HIV RNA. CA-HIV DNA and RNA were measured by qPCR assays in PBMCs and CSF cell pellets derived from approximately $13 \mathrm{~mL} \mathrm{CSF}$ and normalized by amplifiable CCR5 cell equivalents using previously described methods (39). Cell-free HIV RNA was quantified by iSCA in CSF supernatant (3-5 $\mathrm{mL}$ ) and blood plasma (5 mL) (25). By limiting dilution analysis, the qPCR assays have been shown to detect 3 copies of HIV-1 DNA or RNA standards in $80 \%$ to $100 \%$ of PCR reactions and 1 copy in $40 \%$ to $70 \%$ of reactions, consistent with Poisson probability distribution for single copy detection (39). Seven inflammatory biomarkers were measured in cell-free CSF and blood plasma by ELISA (IL-6, CXCL-10, CCL2, sCD14, sCD163, TNF- $\alpha$ [all from R\&D Systems], and neopterin [GenWay Biotech]).

Neuropsychological assessment. A 7-domain neuropsychological test battery was administered to the participants with HIV, assessing 
the domains of language/premorbid, fine motor, verbal learning, verbal memory, speed of processing, executive functioning, attention/ working memory. The neuropsychological assessment consisted of 15 individual tests that were administered a median 8 (maximum 35) days prior to or on the same day as the lumbar puncture.

$Z$ scores were calculated by standardizing each raw test score by age, sex, race, and years of education. A deficit score (DS), a measure of neurocognitive impairment, was calculated for each individual test based on the $Z$ score. DSs range from 0 to 5 , with higher scores indicating more severe impairment. Individual DSs were then averaged for an overall GDS, which was clinically validated (20).

Statistical analysis. Rank-based correlations (Spearman) were performed, where results below assay limits were assigned the lowest rank. Groups were compared using the exact Wilcoxon ranksum test for continuous variables and the Fisher's exact test for categorical variables. Regression analyses were used to assess whether associations were influenced by potential confounders. All $P$ values and confidence intervals presented are unadjusted for multiple comparisons performed.

Study approval. The institutional review boards at all participating institutions approved the study. Written informed consent was received from all of the participants enrolled in the study.

\section{Author contributions}

All authors contributed to the data interpretation and writing of this manuscript. All authors, except AV, contributed to the study development, implementation, and analysis.

\section{Acknowledgments}

We sincerely thank the study participants, staff at the ACTG study sites, the ACTG Longitudinal Linked Randomized Trials (ALLRT) team who established the original cohort, the University of Pittsburgh Virology and Immunology Specialty labs, the Yale HARC study staff, the Statistical and Data Analysis Center, and members of the A5321 team. See the Supplemental Acknowledgments for A5321 team details. This work was supported by the National Institute of Allergy and Infectious Diseases and the National Institute of Mental Health of the National Institutes of Health, under award numbers UM1 AI068634, UM1 AI068636, UM1 AI106701, UM1 AI069481, and R21MH110260.

Address correspondence to: Serena Spudich, Department of Neurology, Yale University, 300 George Street, Room 8300c, New Haven, Connecticut 06511, USA. Phone: 203.688.5303; Email: serena.spudich@yale.edu.
1. Churchill MJ, Deeks SG, Margolis DM, Siliciano RF, Swanstrom R. HIV reservoirs: what, where and how to target them. Nat Rev Microbiol. 2016;14(1):55-60.

2. Gray LR, Roche M, Flynn JK, Wesselingh SL, Gorry PR, Churchill MJ. Is the central nervous system a reservoir of HIV-1? Curr Opin HIV AIDS. 2014;9(6):552-558.

3. Estes JD, et al. Defining total-body AIDS-virus burden with implications for curative strategies. Nat Med. 2017;23(11):1271-1276.

4. Lamers SL, et al. HIV DNA Is frequently present within pathologic tissues evaluated at autopsy from combined antiretroviral therapy-treated patients with undetectable viral loads. J Virol. 2016;90(20):8968-8983.

5. Navia BA, Jordan BD, Price RW. The AIDS dementia complex: I. Clinical features. Ann Neurol. 1986;19(6):517-524.

6. Heaton RK, et al. HIV-associated neurocognitive disorders persist in the era of potent antiretroviral therapy: CHARTER Study. Neurology. 2010;75(23):2087-2096

7. Valcour V, Sithinamsuwan P, Letendre S, Ances B. Pathogenesis of HIV in the central nervous system. Curr HIV/AIDS Rep. 2011;8(1):54-61.

8. Yilmaz A, et al. Cerebrospinal fluid neopterin decay characteristics after initiation of antiretroviral therapy. J Neuroinflammation. 2013;10:62.

9. Edén A, et al. Increased intrathecal immune activation in virally suppressed HIV-1 infected patients with neurocognitive impairment. PLoS ONE. 2016;11(6):e0157160.

10. Edén A, et al. HIV-1 viral escape in cerebrospinal fluid of subjects on suppressive antiretroviral treatment. J Infect Dis. 2010;202(12):1819-1825.

11. Ferretti F, Gisslen M, Cinque P, Price RW. Cerebrospinal fluid HIV escape from antiretroviral therapy. Curr HIV/AIDS Rep. 2015;12(2):280-288.

12. Joseph J, et al. Highlights of the Global HIV-1 CSF Escape Consortium Meeting, 9 June 2016, Bethesda,
MD, USA. JVirus Erad. 2016;2(4):243-250.

13. McArthur JC, et al. Identification of mononuclear cells in CSF of patients with HIV infection. Neurology. 1989;39(1):66-70.

14. Ho EL, Ronquillo R, Altmeppen H, Spudich SS, Price RW, Sinclair E. Cellular composition of cerebrospinal fluid in HIV-1 infected and uninfected subjects. PLOS ONE. 2013;8(6):e66188.

15. Sinclair E, et al. Antiretroviral treatment effect on immune activation reduces cerebrospinal fluid HIV-1 infection. J Acquir Immune Defic Syndr. 2008;47(5):544-552.

16. Sönnerborg A, Johansson B, Strannegård O. Detection of HIV-1 DNA and infectious virus in cerebrospinal fluid. AIDS Res Hum Retroviruses. 1991;7(4):369-373.

17. Steuler H, Munzinger S, Wildemann B, Storch-Hagenlocher B. Quantitation of HIV-1 proviral DNA in cells from cerebrospinal fluid. JAcquir Immune Defic Syndr. 1992;5(4):405-408.

18. Saylor D, et al. HIV-associated neurocognitive disorder--pathogenesis and prospects for treatment. Nat Rev Neurol. 2016;12(4):234-248.

19. Peluso MJ, et al. Cerebrospinal fluid HIV escape associated with progressive neurologic dysfunction in patients on antiretroviral therapy with well controlled plasma viral load. AIDS. 2012;26(14):1765-1774.

20. Carey CL, et al. Predictive validity of global deficit scores in detecting neuropsychological impairment in HIV infection. J Clin Exp Neuropsychol. 2004;26(3):307-319.

21. Oliveira MF, et al. Early antiretroviral therapy is associated with lower HIV DNA molecular diversity and lower inflammation in cerebrospinal fluid but does not prevent the establishment of compartmentalized HIV DNA populations. PLOS Pathog. 2017;13(1):e1006112.

22. Shaunak S, Albright RE, Klotman ME, Henry SC, Bartlett JA, Hamilton JD. Amplification of
HIV-1 provirus from cerebrospinal fluid and its correlation with neurologic disease. J Infect Dis. 1990;161(6):1068-1072.

23. Steuler H, Munzinger S, Wildemann B, Storch-Hagenlocher B. Quantitation of HIV-1 proviral DNA in cells from cerebrospinal fluid. J Acquir Immune Defic Syndr. 1992;5(4):405-408.

24. Anderson AM, et al. Prevalence and correlates of persistent HIV-1 RNA in cerebrospinal fluid during antiretroviral therapy. J Infect Dis. 2017;215(1):105-113.

25. Palmer S, et al. New real-time reverse transcriptase-initiated PCR assay with single-copy sensitivity for human immunodeficiency virus type 1 RNA in plasma. J Clin Microbiol. 2003;41(10):4531-4536.

26. Cillo AR, et al. Improved single-copy assays for quantification of persistent HIV-1 viremia in patients on suppressive antiretroviral therapy. JClin Microbiol. 2014;52(11):3944-3951.

27. Dahl V, et al. Raltegravir treatment intensification does not alter cerebrospinal fluid HIV-1 infection or immunoactivation in subjects on suppressive therapy. J Infect Dis. 2011;204(12):1936-1945.

28. Dahl V, Peterson J, Fuchs D, Gisslen M, Palmer S, Price RW. Low levels of HIV-1 RNA detected in the cerebrospinal fluid after up to 10 years of suppressive therapy are associated with local immune activation. AIDS. 2014;28(15):2251-2258.

29. Farhadian SF, et al. Single-cell RNA sequencing reveals microglia-like cells in cerebrospinal fluid during virologically suppressed HIV. JCI Insight. 2018;3(18):121718.

30. Lamers SL, et al. HIV DNA is frequently present within pathologic tissues evaluated at autopsy from combined antiretroviral therapy-treated patients with undetectable viral loads. J Virol. 2016;90(20):8968-8983.

31. Gelman BB. Neuropathology of HAND with suppressive antiretroviral therapy: encephalitis 


\section{CLINICAL MEDICINE}

and neurodegeneration reconsidered. Curr HIV/AIDS Rep. 2015;12(2):272-279.

32. Klatt NR, Chomont N, Douek DC, Deeks SG. Immune activation and HIV persistence: implications for curative approaches to HIV infection. Immunol Rev. 2013;254(1):326-342.

33. Couturier J, Lewis DE. HIV persistence in adipose tissue reservoirs. Curr HIV/AIDS Rep. 2018;15(1):60-71.

34. Gandhi RT, et al. Levels of HIV-1 persistence on antiretroviral therapy are not associated with markers of inflammation or activation. PLoS Pathog. 2017;13(4):e1006285.

35. Clifford DB. HIV-associated neurocognitive disorder. Curr Opin Infect Dis. 2017;30(1):117-122.

36. Tedaldi EM, Minniti NL, Fischer T. HIV-associated neurocognitive disorders: the relationship of HIV infection with physical and social comorbidities. Biomed Res Int . 2015;2015:641913.

37. Coban $\mathrm{H}$, et al. Impact of aging on neurocognitive
The Journal of Clinical Investigation

performance in previously antiretroviral-naive HIV-infected individuals on their first suppressive regimen. AIDS. 2017;31(11):1565-1571.

38. Kessing CF, et al. High number of activated CD8+ $\mathrm{T}$ cells targeting HIV antigens are present in cerebrospinal fluid in acute HIV infection. J Acquir Immune Defic Syndr. 2017;75(1):108-117.

39. Hong F, et al. Novel assays for measurement of total cell-associated HIV-1 DNA and RNA. JClin Microbiol. 2016;54(4):902-911. 